\title{
La pause : un paramètre variable dans le discours politique
}

\author{
Frédéric André ${ }^{1}$, Hirsch Fabrice ${ }^{1}$, Bechet Marion ${ }^{2}$, Barkat-Defradas Mélissa ${ }^{1}$, Didirkova Ivana ${ }^{1}$, \\ Maturafi Lavie ${ }^{1} \&$ Baklouti Elodie ${ }^{1}$ \\ ${ }^{1}$ Laboratoire Praxiling, UMR 5267-CNRS, Université Paul Valéry, Montpellier \\ ${ }^{2}$ EA1339 LilPa, Equipe Parole et Cognition, Université de Strasbourg \\ fabrice.hirsch@univ-montp3.fr
}

\section{Introduction}

\subsection{La parole politique}

Depuis toujours, la politique développe un monde de représentations. Un ou plusieurs hommes ou femmes représentent en effet des groupes de populations, eux-mêmes représentant tout un ensemble d'idées caractérisant leurs avis sur la société et plus largement leurs souhaits concernant l'avenir de leur nation. Toutes ces formes de représentations servent à organiser les sociétés démocratiques depuis l'Antiquité, en s'appuyant avant tout sur une forme langagière particulière : l'oral. L'oral est effectivement, encore aujourd'hui, la forme langagière principale et privilégiée de l'échange et de la transmission d'idées, connues ou nouvelles, qui peuvent être, par son biais, présentées, expliquées, jaugées, jugées, réfutées, améliorées, ou plus simplement débattues.

La notion de parole politique s'est donc développée très tôt dans l'Histoire, parallèlement aux autres formes de langages oraux, et s'est, au fil du temps, techniquement affinée pour finalement aboutir à la mise en place de formes spécifiques (Gerstlé, 1992). Or, avec le développement des sociétés modernes et la multiplication des supports de diffusion, ces différentes formes se sont détaillées, en fonction des cadres de diffusion, ou encore du nombre de participants à l'échange. Ainsi, plusieurs types de discours ont vu le jour, présentant des formes issues de l'oral, mais travaillées pour participer stratégiquement à l'aboutissement d'un objectif préalablement fixé.

Un même homme politique multiplie donc aujourd'hui les formes que peuvent prendre sa parole publique au sens large, en fonction de son cadre d'énonciation : les modifications peuvent aussi bien porter sur le choix des mots à employer que sur le support de ce contenu, c'est-à-dire la voix et ses caractéristiques prosodiques (Bechet et al., 2013).

L'une des caractéristiques prosodiques essentielles dans le discours politique concerne l'organisation temporelle de la parole, dans la mesure où il s'agit d'un miroir de la relation au pouvoir : l'aspect temporel peut être représentatif de la distance sociale qui existe entre l'homme politique et son audience. Cette distance peut être divisée en quatre catégories majeures : intime, informelle, sociale, et publique (Hall, 1959). L'homme politique adapte donc son élocution en fonction de chacune de ces "distances". C'est la raison pour laquelle Duez (2003) a pu mettre en corrélation certains facteurs prosodiques et des situations d'élocution précises.

\subsubsection{Entretiens amicaux}

Lors d'entretiens amicaux, l'homme politique répond à son interlocuteur de manière informelle : son objectif est ici simplement d'informer et de séduire un électorat potentiel ou acquis (Duez, 2003). Ses réponses spontanées sont donc marquées par de nombreuses pauses pleines (euh, allongements, répétitions non sémantiques), relativement longues. De plus, le débit est généralement rapide : les pauses situées aux frontières syntaxiques (phrases ou propositions) sont donc peu marquées. 


\subsubsection{Entretiens politiques}

A l'inverse, dans les entretiens politiques, les mêmes hommes politiques répondent à des questions sur des événements contemporains, ils interprètent, critiquent et proposent des solutions : il s'agit donc de persuader l'auditeur tout en étant spontané et (relativement) informel (Gauthier, 1994). Dans cette situation, Duez a remarqué que les temps de pauses sont plus importants, mais que les hésitations sont moins nombreuses et plus brèves. Notons également que celles-ci prennent davantage la forme de syllabes allongées que de "euh", dans la mesure où les premières passent plus facilement inaperçues et répondent en effet mieux à l'objectif de séduction et de persuasion du locuteur (Duez, 2003).

\subsubsection{Discours politiques}

Dans les discours politiques, généralement médiatisés (radiophoniques et télévisuels), l'objectif de l'homme politique est tout autre : il cherche à séduire les électeurs, et à les persuader de voter pour lui. Dans ce cadre grave et formel, le contact entre le locuteur et son auditoire est distant et indirect. Selon les relevés de Duez (2003) et de Léon (1971), les stratégies prosodiques mises en place sont donc les suivantes : la vitesse d'élocution est lente, et le rythme régulier tend vers le rythme ternaire de l'alexandrin français. Paradoxalement, bien que le contenu soit connu et qu'il n'y ait aucune improvisation dans le discours, le temps de pause est nettement supérieur (plus de $50 \%$ ) à celui relevé dans les entretiens politiques et dans les entretiens amicaux. La distribution des types de pauses interlexicales est également très différente d'une prosodie "classique". Bien que les pauses servant à la démarcation syntaxique soit toujours majoritaires, l'utilisation des pauses de focalisation est privilégiée puisqu'elles permettent d'attirer l'attention sur le mot ou l'expression qui suit et l'idée qu'ils transmettent. Ce type de pauses, qui sont de véritables brèches dans le discours, sont, dans ce cadre, une des composantes essentielles de la parole politique au sens large. En effet, on les retrouve dans les allocutions d'homme politiques français, mais également américains (Carter et Reagan) et allemands (Schmidt et Strauss) (Duez, 2003).

\subsection{Objectifs et hypothèses}

Dans cette recherche, nous avons fait le choix d'étudier la parole de François Hollande dans différents contextes de la campagne électorale de 2012, à savoir durant le débat du second tour de la Primaire Citoyenne, lors du discours du Bourget, où François Hollande était simple candidat, et au moment du discours qu'il a prononcé à l'Hôtel de Ville de Paris en tant que nouveau Président de la République française.

Notre objectif était d'étudier l'emploi de la pause dans la parole des hommes politiques, à travers l'étude de la parole de François Hollande, et d'observer si des variations sont notables dans l'utilisation des silences dans le discours de ces derniers.

Pour répondre à cet objectif, cette étude se fonde sur les travaux menés par Duez $(1999$; 2003), qui ont révélé que la durée des pauses varie en fonction de la situation de l'homme politique vis-à-vis du pouvoir (dans l'opposition vs. au pouvoir) et par rapport aux thèmes abordés durant un débat. Danielle Duez a pu mettre en lumière des points précis relatifs à l'organisation prosodique mise en place par plusieurs hommes politique français influents, tels que Jacques Chirac, Lionel Jospin, ou encore François Mitterrand. L'homme politique doit en effet faire face à de nombreux paramètres lorsqu'il s'exprime, que ce soit de manière informelle dans un entretien amical, ou devant un large public, lors d'un discours de campagne électorale, par exemple. Ainsi, il doit adapter sa prosodie à la situation de communication, et aux attentes de l'auditeur.

Dans une analyse comparative de trois discours de François Mitterrand à différents moments de sa carrière, Duez a pu mettre en exergue un lien entre l'organisation temporelle de la parole politique et la situation au pouvoir du locuteur. Selon ses observations, «la vitesse d'élocution est significativement 
plus lente dans les discours présidentiels que dans les discours d'opposant. De plus, les temps de pause de ces discours sont significativement inférieurs au temps de pause d'un discours présidentiel», du fait de l'allongement de la durée moyenne du temps de chaque pause. Ces constatations sont confirmées par les observations réalisées sur des discours où l'homme politique est à la fois président sortant et candidat à l'élection. Il semble donc qu'une organisation temporelle différente est mise en place dans chacune des situations étudiées. Lorsqu'il est opposant (1974), François Mitterrand accélère sa vitesse d'élocution, marquant des pauses brèves, afin d'accumuler un maximum d'arguments dans un temps de parole court. A l'inverse, lorsqu'il est le président en place (1984), sa vitesse d'élocution est lente, et il aménage un grand nombre de pauses plus longues que la moyenne aux frontières syntaxiques, lui donnant un effet de stature stable et réfléchie. Enfin, dans la situation intermédiaire (1988), ou il est à la fois président sortant et candidat à l'élection présidentielle, les données sont, comparées aux deux extrêmes vus précédemment, intermédiaires. "Il peut, dans sa situation, se contenter de rappeler son bilan ; mais en tant que candidat, il se doit aussi de persuader l'électorat et d'exploiter le temps qui lui est imparti pour développer son argumentation. Il essaie donc de réduire la distance entre les deux rôles et se conduit bien en présidentcandidat » (Gerstlé, 1992).

Afin de poursuivre ces recherches, notre étude vise à vérifier si l'utilisation et les caractéristiques de la pause varient en fonction de la nature du débat. Notre hypothèse est que l'homme politique adapte le rythme de sa parole, et donc les pauses présentes dans ses énoncés, en fonction de son contradicteur et du type de débat.

Pour vérifier cette assertion, nous avons fait le choix d'étudier les pauses chez François Hollande, d'abord en situation de débat face à Martine Aubry, durant la primaire socialiste de 2011, puis en situation de discours, lors du discours préélectoral prononcé au Bourget.

La comparaison de ces deux situations est particulièrement intéressante dans la mesure où il s'agit de deux formes de discours très proches et en même temps très éloignées. En effet, dans les deux cas, l'objectif premier du locuteur est de convaincre le public par la transmission de ses idées. Cependant, dans le cas du discours, celui-ci est écrit, préparé et répété par le locuteur qui s'adresse directement au public sans risque de se faire couper la parole ou devoir répondre à des questions qui peuvent être délicates. Dans le cas du débat, le locuteur s'adresse au public, mais à travers un discours qu'il émet vers son interlocuteur. Là encore, le discours est préparé, mais il n'est pas forcément évident de l'exprimer librement puisqu'il doit s'adapter à la situation de communication du débat.

Ainsi, nous verrons dans cette étude l'étendue de variation existant dans la gestion des pauses qui, de fait, sera différente dans chacun des cas.

\section{Méthodologie}

L'étude porte sur deux types d'exercice de communication : un discours et un débat.

Le débat correspond au final de la Primaire socialiste qui s'est tenu le 12/10/2011, où FH était opposé à Martine Aubry pendant une durée totale de 1h27'50". Diffusé en direct et en prime time sur France 2, ce débat fut modéré par 4 journalistes, en fonction des différentes thématiques abordées : Fabien Namias pour l'économie, Patrick Cohen pour les questions sociales, Françoise Fressoz pour parler de l'Europe et du Monde, en enfin David Pujadas, modérateur principal, pour aborder les questions de méthodes et comparer les types de présidences potentielles. Les débatteurs sont assis de trois quart, face aux journalistes, et entourés d'un public inaudible. FH porte un costume fonce, veste ouverte, ainsi qu'une cravate bleue, nouée en V. Il est assis en bout de chaise, dans une posture assez naturelle. Il est à l'aise, expressif et démonstratif. Son attention est ainsi multi-focalisée, entre les journalistes qui l'interrogent, son adversaire, Martine Aubry, et le contact visuel direct avec la camera/le téléspectateur. S'agissant d'un débat, FH ne s'exprime pas sur l'ensemble du document. Son temps de parole, pauses comprises, est donc de 39'28". 
Le second document présente la vidéo d'un meeting de FH qui s'est tenu le 22/01/2012, au Bourget. Il s'agit d'un discours d'une durée de $1 \mathrm{~h} 25^{\prime} 42^{\prime \prime}$, diffusé en direct, au cours de la journée. FH se présente seul au pupitre, et prononce un discours préalablement écrit, le texte est placé devant lui, sans aucun modérateur ou intervenant. Il est donc situé en position centrale, debout, face au large public d'une salle comble. Il porte un costume fonce, dont la veste est ouverte, ainsi qu'une cravate bleue foncé, nouée en W. Tout au long de son discours, il reste debout, gardant néanmoins une posture plutôt naturelle. Très expressif et démonstratif, notamment dans les moments clés de son discours, il cherche à paraitre à l'aise, focalisant son attention sur de nombreuses cibles.

Afin de pouvoir comparer les deux type de production de la parole de façon rigoureuse, les interventions ont été ramenées a une même durée totale. Ainsi, seul un extrait de 39'28" du discours du Bourget a été retenu, afin d'obtenir des résultats pouvant être comparés de façon rigoureuse. L'extrait a été choisi aléatoirement, mais constitue $39^{\prime} 28^{\prime \prime}$ de discours ininterrompu : ainsi, les résultats comparés reflèteront avec plus de justesse les différences possibles qui pourront être observées.

Le débat, comme le discours, a donné lieu à une transcription des propos tenus par François Hollande, puis, après examen de la posture du locuteur, nous avons localisé les pauses et quantifié leur durée à l'aide du logiciel Praat. Les pauses ont été retenues lorsqu'elles étaient égales ou supérieures à $200 \mathrm{~ms}$, puisqu'il s'agit du seuil à partir duquel une pause est susceptible d'être correctement décelée par des auditeurs dans un énoncé (Candéa, 2000).

Ces pauses ont ensuite été distinguées selon les 3 formes citées supra : les pauses de démarcation, pauses de focalisation et la pauses d'hésitation (Ferré, 2003), le signal a ainsi été annoté.

D'autres paramètres (vitesse d'articulation, nombre de syllabes par groupe rythmique et durée des pauses), permettant d'observer les variations de rythme, ont également été quantifiés.

\section{Résultats}

\subsection{Etude du rythme en général}

Les deux formes différentes de discours politiques révèlent des rythmes relativement similaires. En effet, la Figure ci-dessous montre que les vitesses d'articulation sont très proches dans les deux situations, bien que FH s'exprime dans un débit légèrement plus rapide dans le cas du discours du Bourget, avec 6,250 syllabes par seconde, contre 6,171 syllabes par seconde pour le débat de la Primaire PS (ET de 1,605 syll/sec). Cette légère différence peut s'expliquer par le relevé de la durée moyenne des séquences sonores, de 1278 millisecondes pour le Bourget (ET de $654 \mathrm{~ms}$ ) et de $1135 \mathrm{~ms}$ pour le débat de la Primaire PS. Les séquences sonores du Bourget sont sensiblement plus longues que celles du débat face à Martine Aubry.

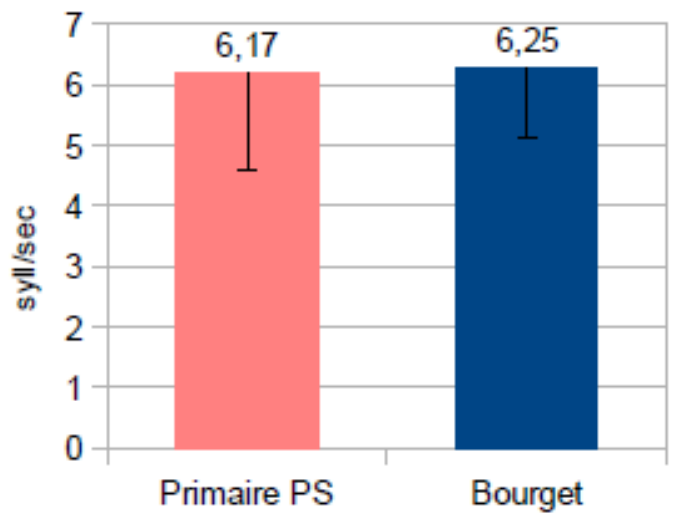


Graphique 1 : Vitesse d'articulation primaire/Bourget

Le ratio de temps de pause/ temps de phonation est de 76,71\% de temps de parole pour 23,29\% de temps de pause lors de la Primaire PS, contre 58,28\% de temps de parole pour $41,72 \%$ de temps de pause lors du discours du Bourget. Des différences assez nettes séparent les deux interventions de FH en termes de gestion du rythme de la parole.

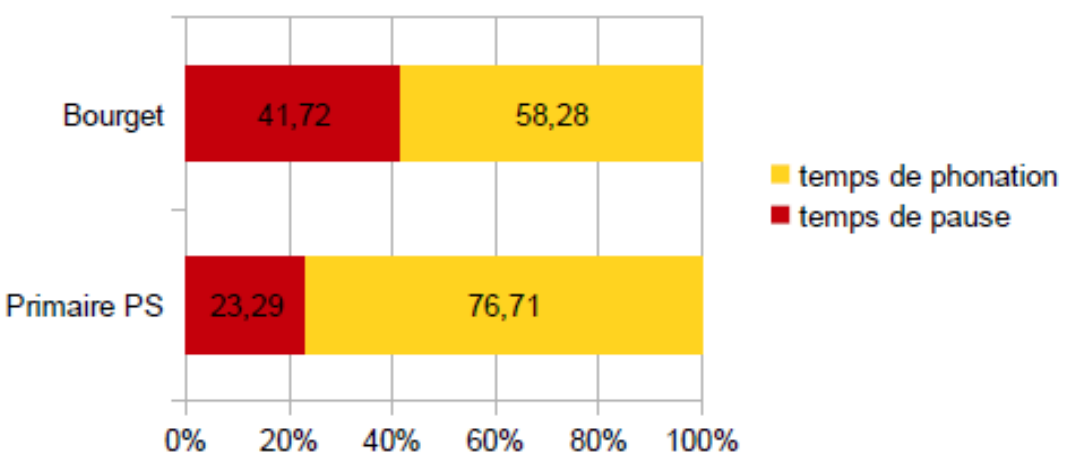

Graphique 2 : Ratio temps de pause/temps de phonation Primaire/Bourget

Les deux interventions étant ramenées à une durée totale équivalente, la comparaison du nombre de pause est rendue possible. Un total de 178 pauses pleines et de 1477 pauses vides ont été comptabilisées lors de la Primaire PS, pour un temps de pause moyen de $373 \mathrm{~ms}$. Concernant le Bourget, 11 pauses pleines (Figure 3) et 1074 pauses vides ont été comptées, d'une durée moyenne de 917 ms (Figure 4).

Des différences très marquées apparaissent entre ces deux interventions, au niveau du nombre total de pauses, du ratio du type de ces pauses, et de leur durée moyenne. Le nombre de pauses est beaucoup plus important dans le cadre du débat de la Primaire citoyenne, surtout les pauses pleines Aussi, la durée moyenne des pauses vides est plus longue de $245 \%$ lors du discours au Bourget.

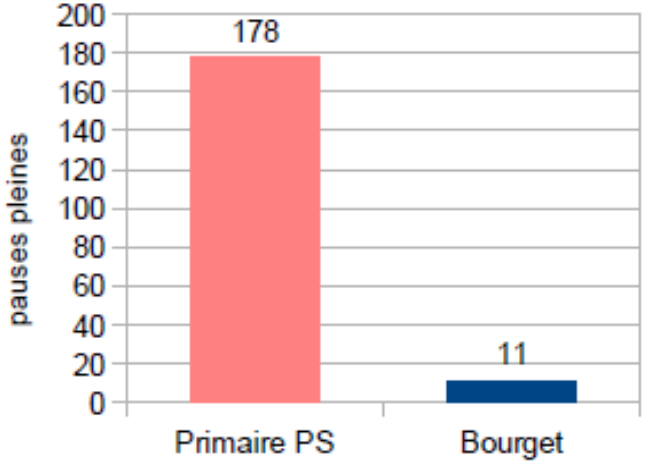

Graphique 3 : Nombre total de pauses pleines

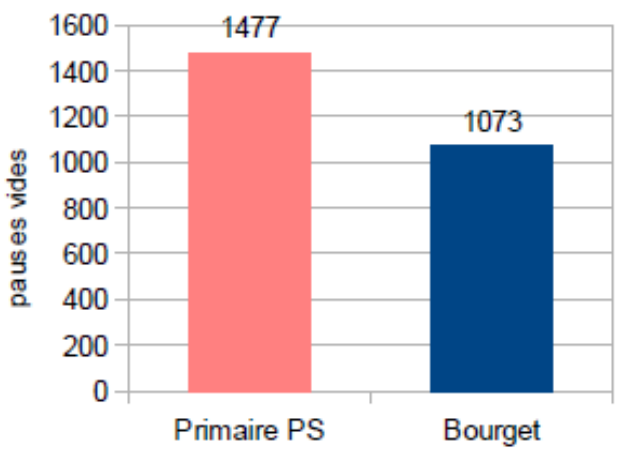

Graphique 4 : Nombre total de pauses vides

Dans le débat des Primaire PS, on rencontre dans la parole de FH un grand nombre de pauses d'une durée relativement courte, en comparaison avec le discours prononcé au Bourget, qui se compose de pauses moins nombreuses mais plus longues, pour finalement obtenir une durée totale de temps de pause presque deux fois plus longue (1,84 fois plus longue). Il est donc intéressant d'analyser plus en détails les modifications rythmiques apportées par FH aux pauses vides dans ces divers cadres. 


\subsection{Etude des pauses en particulier}

Le Graphique 5 illustre les pauses dans la parole de FH lors de son discours au Bourget, légèrement moins nombreuses, mais significativement plus longues que celles de la Primaire Ps, leur durée fluctuant même de façon beaucoup plus importante. Dans le cas du Bourget, précisons que seules les pauses vides d'une durée inférieure à 10 secondes ont été prises en compte pour le calcul des durées moyennes de pause, afin de ne pas fausser les résultats, le discours étant réalisé face à un large public qui parfois empêchait FH de continuer à s'exprimer.

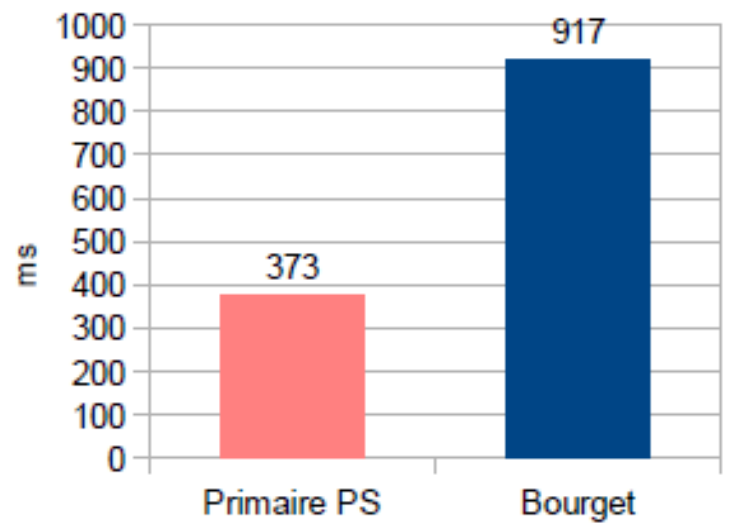

Graphique 5 : Durée moyenne des pauses

Lors du débat face à Martine Aubry, on dénombre FH 1010 pauses de démarcation syntaxique d'une durée moyenne de $392 \mathrm{~ms}$ tandis que son discours prononcé au Bourget en contient 789, d'une durée moyenne de $1014 \mathrm{~ms}$.

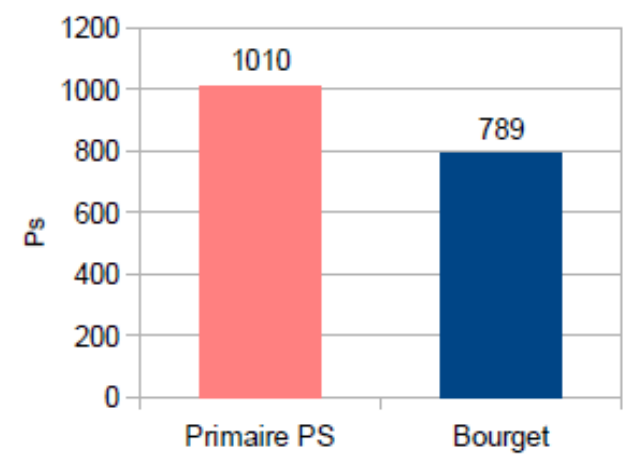

Graphique 6: Nombre total de pauses démarcatives

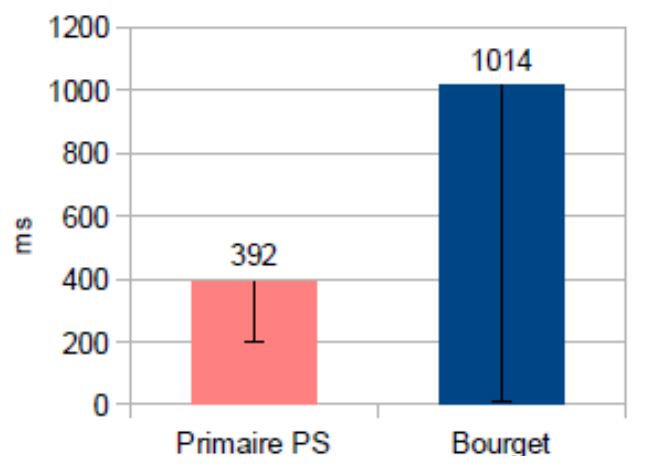

Graphique 7 : Durée moyenne des pauses

Les pauses de focalisation sont, elles, au nombre de 213 pour la Primaire citoyenne, d'une durée moyenne de $315 \mathrm{~ms}$, et de 253 lors du discours du Bourget, d'une durée moyenne de $620 \mathrm{~ms}$. Ici encore, comme le montre la Figure 34, le nombre de pauses de focalisation est beaucoup plus élevé lors du discours du Bourget. La Figure 35 indique une durée moyenne de ce type de pause également plus longue lors du discours au Bourget, avec un écart type plus de 5 fois plus important que lors du débat final de la Primaire PS, suggérant ainsi une variabilité plus élevée dans ce type d'élocution. 


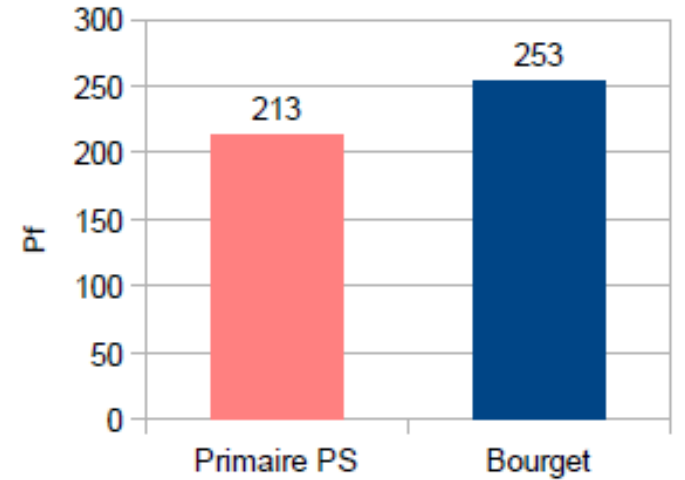

Graphique 8: Nombre total de pauses de focalisation

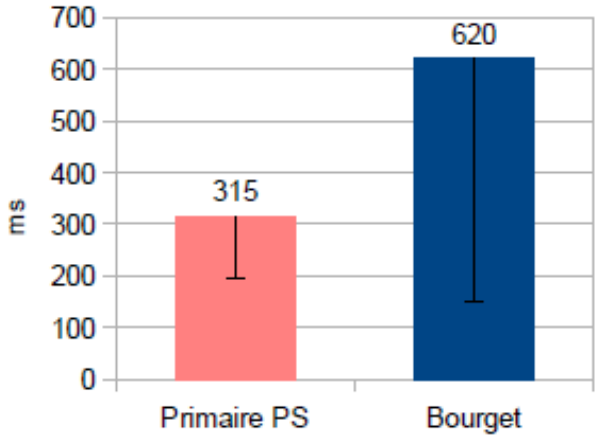

Graphique 9: Durée moyenne des pauses de focalisation

Concernant les pauses d'hésitation, elles sont au nombre de 232 pour une durée moyenne de $341 \mathrm{~ms}$ dans le cadre du débat de la Primaire PS, contre 27 pour le discours du Bourget, d'une durée moyenne de 422 $\mathrm{ms}$ (avec un écart type de $316 \mathrm{~ms}$ ). La comparaison est illustrée infra, dans les Figures 10 et 11 . Les pauses d'hésitation sont bien plus nombreuses dans le débat de la Primaire PS, bien que leur durée moyenne soit sensiblement plus courte. La différence d'écart type, également significative, montre une modulation des pauses d'hésitation lors du discours au Bourget beaucoup plus importante.

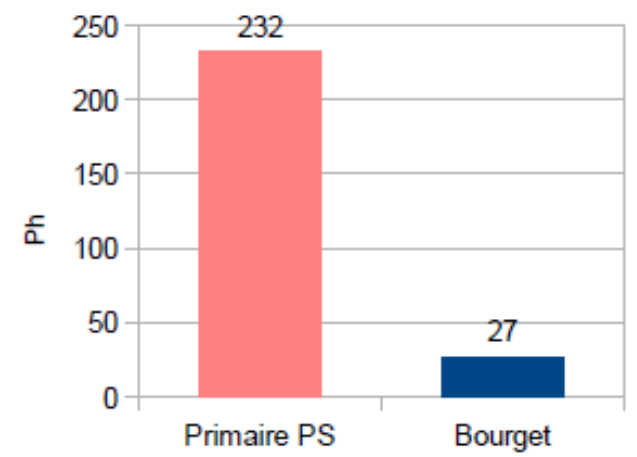

Graphique 10: Nombre total de pauses d'hésitation

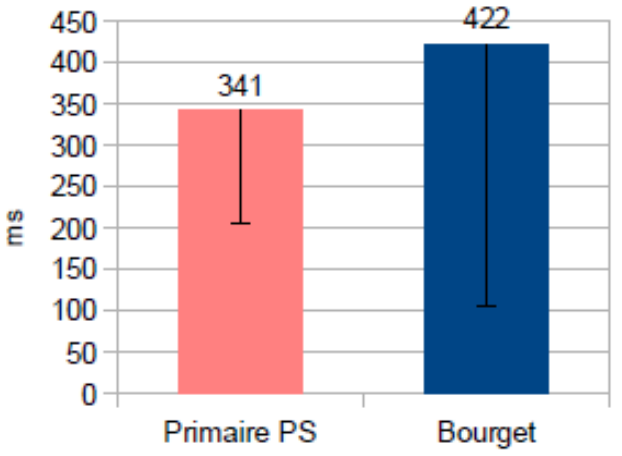

Graphique 11: Durée moyenne des pauses d'hésitation

Notons encore que le nombre de pauses démarcatives décalées est près de trois fois plus important dans le débat de la Primaire PS que le discours du Bourget. Aussi, ces silences sont significativement plus longs que les pauses démarcatives décalées apparaissant dans le discours de FH lors du débat de la Primaire PS. Ces variations peuvent signifier que FH cherche à mettre en place un effet particulier. 


\section{Conclusions}

Globalement, la comparaison des données rythmiques des types de production de parole, en débat et discours préélectoraux, ne montre pas de différences remarquables. En effet, même si la vitesse d'articulation, la durée moyenne des séquences sonores et le nombre moyen de syllabes par séquence sont supérieurs lors du discours du Bourget, les différentes valeurs restent proches les unes des autres.

Dans les deux formes de discours, FH cherche à montrer un profil de leader, supérieur à celui de ses adversaires. On peut néanmoins observer un écart important si l'on considère parallèlement les durées globales de phonation et de pauses des deux interventions. En effet, sur ce point, le calcul du ratio temps de phonation/temps de pause du Bourget montre une durée de temps de pause très importante en comparaison de celle du débat de la Primaire PS, avec une différence de 18,4\% du temps global de l'intervention en pause.

Cette principale distinction est directement liée à la variation dans l'utilisation des pauses, et particulièrement les pauses vides. C'est avec cette variété de pauses que FH va réellement jouer pour créer une différence prosodique, mettant alors en place deux formes distinctes de communication.

Il apparait que le nombre de pauses intradiscursives est globalement plus important dans l'intervention faite lors du débat de la Primaire PS, que ce soit en termes de pauses vides ou pleines.

Cela s'explique par la différence de type de discours. En effet, bien que le débat ait été préparé par FH, que les questions posées et les pièges tendus par son adversaire ou les journalistes soient préalablement déduits des propres "failles" du programme qu'il souhaite présenter, et par conséquent que les réponses sont travaillées et apprises, le niveau de spontanéité reste plus important dans le cadre du débat que lors du discours. FH se contente dans ce cadre-ci de lire le texte de son discours, et, ayant travaillé particulièrement son interprétation, il est normal d'y trouver un nombre de pauses pleines significativement plus faible.

La durée moyenne des pause est en revanche près de deux fois plus élevée pour le discours du Bourget, expliquant, malgré un nombre total de pauses plus faible, que le ratio de temps de phonation/temps de pause présente un pourcentage beaucoup plus important de temps de pause dans ce cadre que dans le celui du débat de la Primaire PS. La combinaison de ces différences constitue un premier point pertinent relatif à la distinction des deux types de discours comparés ici, elle permet d'expliquer la différence de ratio temps de phonation/temps de pause. La comparaison de chaque type de pauses vides appuie encore les différences entre les deux types de discours étudiés. En effet, ces données présentent logiquement des pauses vides plus nombreuses et d'une durée moindre dans le débat de la Primaire PS, mais en ne considérant que les pauses de démarcation syntaxique et d'hésitation. Les pauses de focalisation sont quant à elles plus nombreuses dans le discours du Bourget, et toujours d'une durée moyenne supérieure à celles marquées par FH dans le cadre du débat.

Il semble que FH met davantage en valeur ses propos dans le type de discours relatif au discours préélectoral que dans le débat. Par sa stratégie de parole, FH peut marquer les esprits avec les diverses idées développées. Or, dans le cadre du Bourget, la lecture du texte de son discours permet la mise en place d'une forme de jeu d'acteur, qui favorise le marquage de pauses de focalisation.

De plus, tel que nous l'avons vu supra, lors du débat de la Primaire PS, les deux débatteurs appartiennent à la même classe politique, et se "battent" non pas pour défendre leurs idées au sens large, mais pour défendre leurs moyens et principes organisationnels personnels qui permettent la mise en place de ces idées. Cela peut donc expliquer la différence de pauses de focalisation dans le deuxième cadre d'intervention, puisqu'au Bourget, FH défend seul ses idées (et celles de sa classe politique) en tant que membre du parti majoritaire de l'opposition, face aux autres partis et politiques associés, qui développent parfois des principes contraires aux siens. Il met davantage d'éléments importants pour lui en exergue, puisqu'indissociables de l'ensemble de sa ligne politique. 
On peut néanmoins ajouter que FH a produit au cours de débats de ces élections présidentielles des exemples de longues tirades sans doute préparées au moins en partie, notamment dans le cadre du débat de l'entre-deux tour face à Nicolas Sarkozy ("Moi, Président de la République [...]", en réponse à la question de la journaliste Laurence Ferrari "FH, quel Président comptez-vous être ?"), mais il était alors seul opposant face au Président sortant. Ce type de tirade pourrait faire l'objet d'un type de discours particulier.

L'organisation temporelle d'un discours d'opposant, illustré ici par l'intervention de FH au Bourget, comparée au rythme de la parole mis en place lors du débat politique lors de la Primaire PS, se caractérise donc par une réduction du nombre de pauses marquées, mais une augmentation notable de leur durée moyenne, et d'une augmentation de la proportion de pauses de focalisation marquées.

Ainsi, la stratégie déployée par le locuteur face à la variété de pauses possibles est nettement différente dans les deux cas; celles-ci ponctuent bien différemment chacun des discours prononcés par François Hollande.

\section{Références bibliographiques}

Béchet M., Sandré M., Hirsch F., Richard A., Marsac F., Sock R. (2013) De l'utilisation de la pause silencieusedans le débat politique télévisé. Le cas de François Hollande. Revue Mots. Les langages du politique, 103, 23-38.

Candea M. (2000) Contribution à l'étude des pauses silencieuses et des phénomènes dits 'd'hésitation' en français oral spontané. Étude sur un corpus de récits en classe de français, thèse de doctorat nouveau régime, Université de la Sorbonne nouvelle - Paris III.

Duez D. (2003) Le pouvoir du silence et le silence du pouvoir : comment interpréter le discours politique. MediaMorphoses, no. 8. 2003, p. 77-82.

Duez, D. (1999) La fonction symbolique des pauses dans la parole de l'homme politique. Faits de langues, vol. 13. 1999, p. 91-97.

Ferré G. (2003) Les pauses démarcatives déplacées en anglais spontané, Lidil, 26:155-169, 2003.

Ferré G. (2004) Les pauses intra-constituant en anglais spontané, XXVemes Journees d' Etude sur la Parole, Fes.

Gauthier, G. (1994). Les débats politiques télévisés: propositions d'analyse. Les Études de communication publique, 9. Québec : Université Laval

Gerstlé J. (1992) La communication politique, Que sais-je.

Goldman-Eisler F. (1968) Psycholinguistics. Experiments in spontaneous speech. London and New York : The Academic Press.

Goldman-Eisler F., Cohen M. (1972) An Experimental Study of Interference Between Receptive and Productive Processes Relating to Simultaneous Translation 
Hall E.-T. (1959) The silent language, editions Doubleday and Cie, New York.

Léon, L. (1971). L'art oratoire du président de Gaulle, Essais de phonostylistique, Studia Phonetica, Montréal, Paris, Bruxelles : Didier, 131-144.

Maclay H. \& Osgood C.E., (1959) Hesitation Phenomena in Spontaneous English Speech, Word, n¹1.

Sacks H., Schegloff E. A., Jefferson G. (1974) «A Simplest Systematics for the Organization of Turn-Taking for Conversation », Language, $\mathrm{n}^{\circ} 50,4$, p. 696-735.

Simon A. C., Auchlin A., Avanzi M. \& Goldman J.-P. (2010) Les phonostyles. Une description prosodique des styles de parole en français. In Abecassis, M. \& G. Ledegen (éds), Les voix des Français, vol. 2. En parlant, en écrivant (= Actes du colloque AFLS «Les voix du français : usages et représentations », Taylorian Institution, The University of Oxford, 3-5 septembre 2008), Berne: Peter Lang, pp. 71-88.

Zellner B., (1994) Pauses and the temporal structure of speech, Fundamentals of speech synthesis and speech recognition, editions E. Keller. 